\title{
Design, Validation and Reproducibility of a Short Food Frequency Questionnaire to Assess Fruit and Vegetable Intake in Schoolchildren from Northwest Mexico
}

\author{
Trinidad Quizán-Plata ${ }^{1,}$, , Julián Esparza-Romero², Adriana Verónica Bolaños-Villar², \\ María Guadalupe Corella Madueño ${ }^{3}$, Antonio Rascón Careaga ${ }^{3}$ \\ ${ }^{1}$ Nutritional Sciences Coordination, Department of Chemical and Biological Sciences, Division of Biological and Health Sciences, \\ University of Sonora, Hermosillo, Mexico \\ ${ }^{2}$ Department of Health and Public Nutrition, Nutrition Coordination, Center for Food Research and Development, Hermosillo, Mexico \\ ${ }^{3}$ Department of Chemical and Biological Sciences, Division of Biological and Health Sciences, University of Sonora, Hermosillo, Mexico
}

\section{Email address:}

tquizan@guayacan.uson.mx (T. Quizán-Plata),julian@ciad.mx (J. Esparza-Romero), avb@ciad.mx (A. V. Bolaños-Villar), lcorella@guayacan.uson.mx (M. G. Corella-Madueño), arascon@guayacan.uson.mx (A. Rascón-Careaga)

${ }^{*}$ Corresponding author

\section{To cite this article:}

Trinidad Quizán-Plata, Julián Esparza-Romero, Adriana Verónica Bolaños-Villar, María Guadalupe Corella Madueño, Antonio Rascón Careaga. Design, Validation and Reproducibility of a Short Food Frequency Questionnaire to Assess Fruit and Vegetable Intake in Schoolchildren from Northwest Mexico. International Journal of Nutrition and Food Sciences. Vol. 5, No. 5, 2016, pp. $337-343$. doi: $10.11648 /$ j.ijnfs.20160505.14

Received: August 20, 2016; Accepted: September 2, 2016; Published: September 21, 2016

\begin{abstract}
In Mexico, the 2012 National Health and Nutrition Survey estimated that $65.6 \%$ of the schoolchildren aged 5-11 years has inadequate intake of fruits and vegetables. This study was conducted to validate and determine the reproducibility of a short Food Frequency Questionnaire (FFQ) designed to measure changes in fruit and vegetable intake in schoolchildren from Northwest Mexico. To the development of the FFQ, a total of 249 Dietary Recalls (24HR) were collected from 189 schoolchildren. To evaluate validity and reproducibility, two non-consecutive 24HR (as reference method) and two FFQ were collected from 124 schoolchildren. They were randomly divided into two groups: intervention (62) used for reproducibility and control (62) to evaluate validity. The intervention group received a program that promotes consumption of fruits and vegetables for a period of 6 months. Spearman correlations, Wilcoxon test and Bland-Altman plots were used to assess validity. Reproducibility statistics included Spearman correlations, Percent agreement and Weighted Kappa ( $\kappa w)$ test and Bland-Altman plot. For validity, the mean intake estimated did not differ significantly for vegetable and the combined vegetable and fruit but it was different in fruit intake. There was a moderate correlation between FFQ1 and the 24HR to estimate the consumption of fruit or vegetable $(r=0.38, r=0.48)$ and improves for fruit and vegetables combined $(r=0.69)$ and the bland and altman plot, showed a homogeneous dispersion of points around the line of the differences for the consumption of fruit and vegetable. For FFQ reproducibility the spearman coefficient values ranged from 0.47 for fruit intake to 0.73 for vegetable and fruit combined intake. Cross-classification between the two methods showed that $>30 \%$ of subjects were classified correctly and the $\mathrm{kw}$ ranged between 0.425 for fruit to 0.605 for vegetable and fruit, indicating from moderate to substantial level of agreement. The results on validation and reproducibility indicate that the two methods (FFQ and 24HR) give similar results. Food frequency questionnaire has validity and reproducibility to evaluate intake of fruit and vegetable in schoolchildren from northwest Mexico, and can be used to evaluate changes in fruit and vegetable intake on intervention programs, specially aimed to increase the consumption of such food.
\end{abstract}

Keywords: Food Frequency Questionnaire, Validity, Fruit and Vegetable Intake, Schoolchildren, Northwest Mexico 


\section{Introduction}

In September 2004, WHO office In Kobe Japan held the first workshop on "fruits and vegetables for health", led by experts from the sectors of nutrition, agriculture and health and other institutions. The objective of this workshop was the improvement of working parameters to guide the development of interventions promoting fruit and vegetables consumption in the world. Two of the conclusions of the workshop included recommendations for further work to promote the intake of fruits and vegetables, involving the development of intervention models, especially in developing countries and, the improvement of simple tools to measure the effectiveness of such interventions, as short food frequency questionnaires [1].

The World Health Organization (WHO) suggests a daily intake of $400 \mathrm{~g}$ of fruits and vegetables to prevent chronic diseases such as heart disease, cancer, diabetes or obesity; as well as to prevent and mitigate micronutrient deficiencies [2]. However, the estimated current consumption of fruits and vegetables is highly variable around the world, ranging from $100 \mathrm{~g}$ per day in less developed countries to about $450 \mathrm{~g}$ per day in Western Europe [3].

In Mexico, the 2006 National Health and Nutrition Survey estimated that only $30 \%$ of Mexican population has an adequate intake of fruits and vegetables, and only $17 \%$ of the schoolchildren aged 5-11 years, adhere to the international recommendations [4]. Therefore, Mexico have launched some nutritional public policies on healthy food products; including the National Strategy against Overweight and Obesity. One of the objectives of this program is to promote the design of intervention programs to increase the daily consumption of at least 5 fruits and vegetables in schoolchildren [5]. Public Health Institutions have being doing some efforts to meet the recommendations. Thus, the results of the National Survey of Health and Nutrition 2012, demonstrated that the consumption of fruits and vegetables was $34.4 \%$ in the schoolchildren [6]. Therefore, it is important to consider the suggestion of WHO of having valid and reproducible dietary tools that can accurately determine changes in the consumption of fruits and vegetables, especially when interventions aimed to increase its consumption. The food frequency questionnaire (FFQ) is a tool suggested by epidemiologists to explore changes over time in food consumption because it is cheap, easy and quick to apply. One of the advantage of FFQ is that requires less effort by the respondent than other methods, it does not alter the usual pattern of consumption and the variation of interindividual intake is lower even with a single interview of FFQ when compared with a single application of a $24 \mathrm{HR}$. The FFQ is able to classify individuals by its consumption allowing comparisons and identifying high-risk behaviors [7]. Although some studies indicate that consumption of fruits and vegetables among Mexican schoolchildren is limited, there is not an exclusive FFQ to assess its consumption. Ramirez-Silva et al. (2009), reported that consumption of fruits and vegetables among schoolchildren in the northern region of Mexico, is low compared to the center and south regions [8].

Therefore, having a valid and reproducible FFQ that reveals the cultural and seasonal consumption of fruits and vegetables in schoolchildren in Northwest Mexico, is important for carrying out interventions that promote the consumption of fruits and vegetables.

In the international context, there are well validated FFQs, designed to assess fruit and vegetable consumption [9-13]. However, all of these studies have a cross-sectional design. Conversely, our study determined fruit and vegetable consumption after an intervention aimed to increase its consumption. Thus, we wanted to validate the FFQ and to estimate its reproducibility to classified schoolchildren from Northwest Mexico, according to their usual fruit and vegetable intake and the changes in their consumption.

\section{Methods}

\subsection{Study Design and Subjects}

In total, 189 participants were randomly recruited for the study from august 2009 to September 2010 from Northwest Mexico. The participants were first grade students of public primary school aged between 6 to 8 years old including boys and girls. An inclusion criteria used in this study, was to select the participants considering the index of marginalization (IM) where their schools were located. IM is a measure of lack in education, housing services and monetary income; and classifies regions in five categories: very low, low, medium, high and very high. Higher IM indicates lower income, poor housing services and lower education. For this study only areas with very low to medium IM were selected.

After having received verbal and written information, all participants gave written consent about the study. The study was carried out at the Health Sciences Postgrad of University of Sonora and was approved by the local ethics committee.

\subsection{FFQ Development}

To design the FFQ of fruit and vegetable, two nonconsecutive 24 hour dietary recalls (24HR), applied by a trained interviewer were collected from 60 schoolchildren and a 1 day 24HR from 129, making 249 dietary recalls. Food and beverages consumed by schoolchildren during the 24 hours before the interview were registered. The day was divided in six periods (morning, midmorning, lunch time, afternoon, dinner and later in the evening). 24HRs were applied to the child in the presence of his mother. The amount of each food and drink consumed was recorded, regardless of whether or not it was fruit or vegetable, including recipes and preparation form. Cardboard and plastic food models were used to obtain a more accurate quantitative estimate of dietary intake.

From the dietary information of the $24 \mathrm{HR}$, a selection of fruit and vegetable to include them in the FFQ was performed according to the following criteria: a) fresh fruits 
and $100 \%$ pure juice were listed in the fruit group; b) vegetables were considered those fresh or cooked; and c) food consumed by at least $30 \%$ of the population on one week [14].

FFQ was ordered as follow:

Food groups: Selected foods were classified into two groups: 1) fruits and 2) vegetables, and both were sorted alphabetically.

Mean portion: To obtain the mean portion $(0.50)$, the average consumption of each fruit and vegetable was estimated in grams from the nonconsecutive 24HR. The mean portion in the questionnaire was added in terms of numbers of pieces, slices or household measures for quantitative estimation [15].

Size portion: Three categories of portion size were defined: small (S), medium (M) and large (L). Small and large sizes were defined as 0.25 and 0.75 respects to mean portion [16].

Frequency: Five response categories were established: daily (D), weekly (W), monthly (M), yearly (Y) and never (N) according to Block et al. [15].

A total of 36 items constituted the food frequency questionnaire and it was applied to the child's parent or caregiver (Figure 1).

Food Frequency Questionnaire

Subject ID Date

Please think about the past year and answer the following questions.

Please answer our questions about how much of these fruits and vegetables you ate during the past year.

\begin{tabular}{|c|c|c|c|c|c|c|c|c|c|c|c|}
\hline & \multirow[b]{2}{*}{ Mean portion } & \multicolumn{3}{|c|}{ Size } & \multicolumn{5}{|c|}{ Frequency } & \multirow{2}{*}{$\begin{array}{c}\text { Quantity } \\
\text { (g) }\end{array}$} & \multirow{2}{*}{ Code } \\
\hline Food & & $\mathbf{S}$ & $\mathbf{M}$ & $\mathbf{L}$ & $\mathbf{D}$ & $\mathbf{W}$ & $\mathbf{M}$ & $\mathbf{Y}$ & $\mathbf{N}$ & & \\
\hline \multicolumn{12}{|l|}{ Fruit (fruit and fresh juice } \\
\hline Banana & 1 medium & & & & & & & & & & \\
\hline Cantaloupe & $1 / 4$ medium & & & & & & & & & & \\
\hline Grapefruit & $1 / 2$ medium & & & & & & & & & . & \\
\hline Grapes & 15 pieces & & & & & & & & & & \\
\hline Jicama & 1 slice medium & & & & & & & & & & \\
\hline Orange & 1 small & & & & & & & & & & \\
\hline Orange juice (fresh) & 1 medium glass & & & & & & & & & & \\
\hline Papaya & 1 medium slice & & & & & & & & & & \\
\hline Pear & 1 medium & & & & & & & & & & \\
\hline Peeled Mango & 1 small & & & & & & & & & & \\
\hline Pineapple & I slice & & & & & & & & & & \\
\hline Strawberry & 4 medium & & & & & & & & & & \\
\hline Tangerine & 1 small & & & & & & & & & & \\
\hline Watermelon & 1 medium slice & & & & & & & & & & \\
\hline Whole Apple & 1 medium & & & & & & & & & & \\
\hline \multicolumn{12}{|l|}{ Other fruit not listed } \\
\hline & & & & & & & & & & & \\
\hline & & & & & & & & & & & \\
\hline \multicolumn{12}{|l|}{ Vegetable (Fresh or cooked) } \\
\hline Anaheim chili & 1 small & & & & & & & & & & \\
\hline Avocado (California) & $1 / 8$ medium & & & & & & & & & & \\
\hline Broccoli & $1 / 4$ medium & & & & & & & & & & \\
\hline Cabbage & $11 / 2$ tablespoon & & & & & & & & & & \\
\hline Carrot (cooked) & $1 / 2$ medium & & & & & & & & & & \\
\hline Carrot (fresh) & $1 / 2$ medium & & & & & & & & & & \\
\hline Celery & $1 / 2$ medium rod & & & & & & & & & & \\
\hline Coriander (cooked) & 1 sprig & & & & & & & & & & \\
\hline Cucumber & $1 / 3$ medium & & & & & & & & & & \\
\hline Jalapeno pepper (canned) & 3 slices & & & & & & & & & & \\
\hline Lemon (fresh) & $1 \mathrm{small}$ & & & & & & & & & & \\
\hline Lemonade (natural) & 1 medium glass & & & & & & & & & & \\
\hline \begin{tabular}{|l|} 
Lettuce \\
\end{tabular} & 1 leaf & & & & & & & & & & \\
\hline Lime & 1 medium & & & & & & & & & & \\
\hline Onion (white) & 1 single slice & & & & & & & & & & \\
\hline Onion cooked & 1 tablespoon & & & & & & & & & & \\
\hline Radish & 1 small & & & & & & & & & & \\
\hline Red pepper (powder) & 2 tablespoon & & & & & & & & & & \\
\hline Spinach (cooked) & 1 cooking spoon & & & & & & & & & & \\
\hline Tomatoes (fresh) & 1 small & & & & & & & & & & \\
\hline Zucchini (cooked) & 1/2 cooking spoon & & & & & & & & & & \\
\hline \multicolumn{12}{|l|}{ Other vegetable not listed } \\
\hline & & & & & & & & & & & \\
\hline & & & & & & & & & & & \\
\hline
\end{tabular}

Figure 1. Food Frequency Questionnaire for fruit and vegetable. 


\subsection{FFQ Validation and Reproducibility}

To evaluate validity and reproducibility, 124 schoolchildren were chosen. Then, they were randomly divided into two groups: intervention (62) used for reproducibility and control (62) to evaluate validity. Only the intervention group received a program that promotes consumption of fruits and vegetables for a period of 6 months. Data collection period to validity and reproducibility are showed in Figure 2.

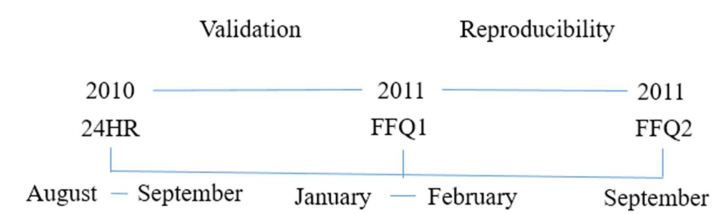

Figure 2. Data collection period for validity and reproducibility.

\subsection{Additional Surveys}

A socio-demographic questionnaire to parents was applied. Marital status, head of the household, level of education of both parents were asked, and whether or not they had medical services. Schoolchildren were measured weight and height in accordance to the internationally standardized procedures. The weight and height were assessed by the WHO Anthro version 1.0.4 Plus program, obtaining the body mass index for age $\mathrm{Z}$ score [17].

\subsection{Data Analysis}

All statistical analyses and calculations were performed using the computer program IBM SPSS statistic version 19 [18].

Data from the first food frequency questionnaire (FFQ1), second food frequency questionnaire (FFQ2) and the estimated from 24 hour recalls were recorded in grams. Differences between the sociodemographic characteristics and anthropometrics data of the participants were assessed using $\mathrm{Chi}^{2}$, Mann-Whitney and independent $\mathrm{T}$ test.

\subsubsection{Validation}

Validity of FFQ was made with dietary data from control group. A comparison of intake's median of fruits, vegetables and total fruits and vegetables from two 24HR and FFQ1 using Wilcoxon test was made. Also, Spearman correlation coefficients were used to measure the strength of the relationship between the consumption of fruits, vegetables and fruits and total vegetables between two 24-hour recalls and FFQ1. Likewise, a Bland-Altman analysis was performed to graphically examine the agreement between the two methods (FFQ1 and 24HR). The plots include lines for the mean difference and the Limits of Agreement (LOA), defined as mean difference $\pm 1.96 \times \mathrm{SD}$

\subsubsection{Reproducibility}

Spearman correlation coefficient was used to assess the reproducibility and the effect of the intervention for correlations between (changes in) dietary intake assessed with the FFQ1 and FFQ2. The degree of agreement between FFQ1 and FFQ2 was further evaluated using contingency tables of tertiles and weighted Kappa ( $\mathrm{\kappa w}$ ) test with values of $\kappa \mathrm{w}$ between 0.40 and 0.59 considered moderate, 0.60 to 0.79 substantial and 0.8 outstanding as per Landis \& Koch [19]. Also, a Bland-Altman plot from the intake of fruit and vegetable from FFQ1 and FFQ2 was used to graphically examine the agreement between FFQ1 and FFQ2.

\section{Results}

\subsection{General Characteristics}

A total of 124 participants completed the FFQ1, FFQ2 and two 24HR. The average age of intervened schoolchildren was 7.2 years (range 6.6-8.5) and 7.1 years of the control group (range 6.6-7.6). Weight, height and BMI/age percentile are shown in Table 1.

Table 1. Anthropometric data of schoolchildren according to study groups before intervention.

\begin{tabular}{llll}
\hline Variables $^{\mathbf{1}}$ & Intervention & Control & P \\
\hline Schoolchildren $(\mathrm{n})$ & 62 & 62 & $-\mathrm{----}$ \\
Age (years) & $7.2 \pm 1.2$ & $7.1 \pm 1.1$ & 0.38 \\
Weight $(\mathrm{kg})$ & $27.17 \pm 7.6$ & $26.25 \pm 6.6$ & 0.32 \\
Height $(\mathrm{cm})$ & $123.28 \pm 6.5$ & $122.66 \pm 5.7$ & 0.46 \\
BMI/age (percentile) & $62.33 \pm 31.8$ & $63.06 \pm 29.3$ & 0.58 \\
\hline
\end{tabular}

${ }^{1} \mathrm{~T}$ test for independent samples

Sociodemographic characteristics of families in both study groups are shown in Table 2 . In both groups the average family size was 5 members. Parents are mostly household responsible and most of them were married. The educational level of parents was quite similar in both groups and more than $85 \%$ of schoolchildren in both groups had free medical service.

Table 2. Sociodemographic characteristics.

\begin{tabular}{|c|c|c|c|c|c|c|}
\hline & & \multicolumn{2}{|c|}{ Intervention } & \multicolumn{2}{|c|}{ Control } & \multirow[b]{2}{*}{$\mathbf{P}$} \\
\hline & & $\mathbf{n}$ & $\%$ & $\mathbf{n}$ & $\%$ & \\
\hline \multirow{3}{*}{$\begin{array}{l}\text { Household } \\
\text { responsible }^{1}\end{array}$} & Father & 30 & 48.4 & 43 & 67.2 & \multirow{3}{*}{0.16} \\
\hline & Mother & 6 & 9.6 & 7 & 10.9 & \\
\hline & $\begin{array}{l}\text { Both, } \\
\text { grandparents }\end{array}$ & 26 & 42 & 14 & 21.9 & \\
\hline \multirow{3}{*}{$\begin{array}{l}\text { Marital status } \\
(\text { mother })^{1}\end{array}$} & Married & 45 & 72.6 & 47 & 73.4 & \multirow{2}{*}{0.91} \\
\hline & Other & 17 & 27.4 & 17 & 26.6 & \\
\hline & Elementary & 7 & 5.8 & 6 & 5.0 & \multirow{5}{*}{0.22} \\
\hline \multirow{4}{*}{$\begin{array}{l}\text { Parents School } \\
\text { level }^{1}\end{array}$} & Jr. High school & 41 & 34.2 & 39 & 32.8 & \\
\hline & Technical career & 13 & 10.9 & 11 & 9.3 & \\
\hline & High School & 28 & 23.3 & 30 & 25.2 & \\
\hline & University & 31 & 25.8 & 33 & 27.7 & \\
\hline \multirow[t]{2}{*}{ Medical service ${ }^{1}$} & Yes & 54 & 87.1 & 55 & 85.9 & \multirow{2}{*}{0.78} \\
\hline & No & 8 & 12.9 & 9 & 14.1 & \\
\hline \multirow{2}{*}{$\begin{array}{l}\text { Family monthly } \\
\text { income (USD) } \\
\text { Family size } \\
\text { (mean) })^{3}\end{array}$} & & \multicolumn{2}{|c|}{$\begin{array}{l}791 \\
(554,1231)\end{array}$} & \multicolumn{2}{|c|}{$\begin{array}{l}681 \\
(475,1187)\end{array}$} & 0.21 \\
\hline & & \multicolumn{2}{|l|}{$5 \pm 1$} & \multicolumn{2}{|c|}{$5 \pm 1$} & 0.9 \\
\hline
\end{tabular}

${ }^{1} \mathrm{Chi}^{2}$ test

${ }^{2}$ Mann-Whitney test

${ }^{3}$ Independent sample T test 


\subsection{FFQ Validation}

The mean intake estimated in the control group with two 24HR and the FFQ1 for only vegetable and the combined vegetable and fruit did not differ significantly. In contrast, the consumption of fruit estimated by both methods was statistically different (Table 3). There was a moderate correlation between FFQ1 and the two 24HR to estimate the consumption of fruit or vegetable and improve of fruit and vegetables combined in the control group (Table 3 ).

Table 3. Fruit and vegetable intake comparison and spearman correlation of 62 schoolchildren from $24 \mathrm{HR}$ and FFQ1 in the control group.

\begin{tabular}{lllll}
\hline Food intake & 24HR & FFQ1 & P & r (24 HR vs. FFQ1) \\
\hline Total Fruit (g) & $291.3(193.7,512.8)$ & $250.4(147.5,383.4)$ & 0.031 \\
Total Vegetable $(\mathrm{g})$ & $85.9(47.6,135.0)$ & $77.8(51.0,132.9)$ & 0.664 \\
Total Vegetable and Fruit $(\mathrm{g})$ & $459.3(254.3,646.7)$ & $470.2(201.0,482.0)$ & 0.573 & $0.48 * *$ \\
\hline
\end{tabular}

For intake comparison we use the Wilcoxon paired test. Data are showed as median (interquartile values) $* \mathrm{P}=0.041, * * \mathrm{P}=0.001$

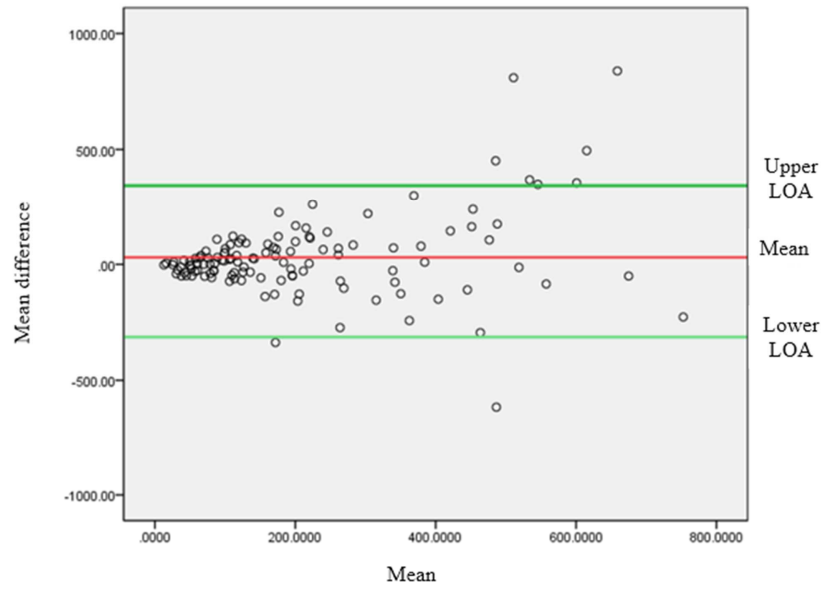

Figure 3. Bland Altman plot showing the relationship between the difference in estimated fruit and vegetable intake of the two methods and the mean estimated fruit and vegetable intake (mean) in the control group (n_62). The upper and lower lines represent the limits of agreement (mean difference \pm $1.96 \times S D)$.

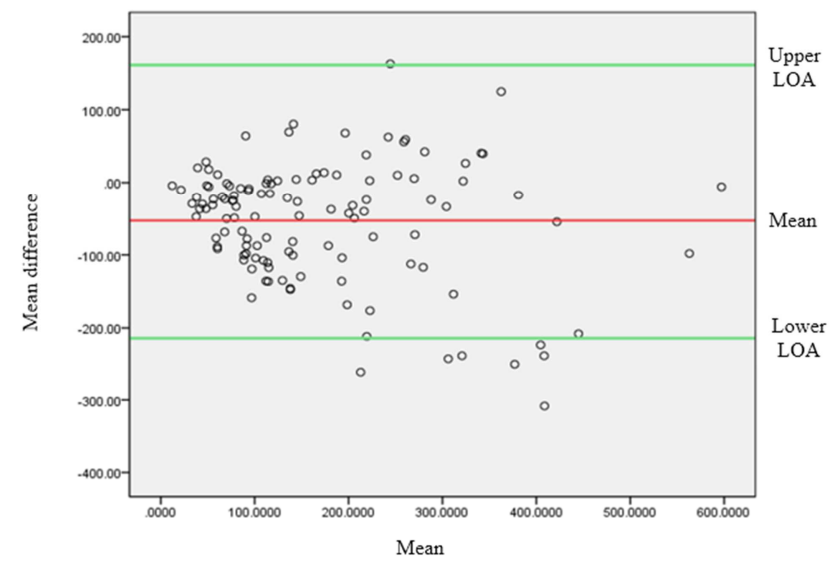

Figure 4. Bland and Altman plot showing the relationship between the difference in estimated fruit and vegetable intake of the FFQ1 and FFQ2 and the mean estimated fruit and vegetable intake (mean) in the intervention group (n_62). The upper and lower lines represent the limits of agreement (mean difference $\pm 1.96 \times S D$ ).

The degree of concordance between the consumption of fruit and vegetable estimated by two $24 \mathrm{HR}$ and the FFQ, showed a homogeneous dispersion of points around the line of the differences for the consumption of fruit and vegetable in the control group (Figure 3). However, the degree of agreement of the consumption of fruit and vegetable in the intervention group analyzed with the FFQ1 and FFQ2, showed a less homogeneous dispersion, with the majority of the points located at the bottom of the line of the differences; nevertheless, they lie within two standard deviations suggested (Figure 4).

\subsection{FFQ Reproducibility}

Regarding the reproducibility of the questionnaire, the Spearman coefficient values ranged from 0.47 for the consumption of fruit to 0.73 for the consumption of vegetable and fruit. All associations were significant (Table 4).

Table 4. Reproducibility (spearman correlation r) from the FFQ1 vs. FFQ2 in the intervention group $(n=62)$.

\begin{tabular}{lll}
\hline Food intake & FFQ1 vs. FFQ2 & P \\
\hline Total Fruit $(\mathrm{g})$ & 0.47 & 0.025 \\
Total Vegetable (g) & 0.49 & 0.001 \\
Total Vegetable and Fruit $(\mathrm{g})$ & 0.73 & 0.001 \\
\hline
\end{tabular}

Table 5, illustrates the reproducibility of the FFQ in terms of absolute agreement (cross-classification of participants by tertiles) between the FFQ1 and FFQ2. The questionnaire classified over $30 \%$ of the participants in the same category of consumption of fruit, vegetable and fruit and vegetable combined, when the FFQ was administered repeatedly. $\mathrm{kw}$ ranged between 0.425 for fruit to 0.605 for vegetable and fruit, indicating a moderate to substantial level of agreement (Table 5).

Table 5. Agreement percentage between FFQ1 and FFQ2 and weighted kappa ( $\kappa w)$ in the intervention group $(n=62)$.

\begin{tabular}{lllll}
\hline Food (g) & T1* & T2* & T3* & Kw \\
\hline & \% & \% & value & \\
\hline Fruit & 75 & 30 & 80 & 0.425 \\
Vegetable & 50 & 45 & 95 & 0.450 \\
Vegetable and fruit & 60 & 40 & 95 & 0.605 \\
\hline
\end{tabular}

* T1, T2 and T3 correspond to tertiles of consumption 


\section{Discussion}

This study was conducted to validate and determine the reproducibility of a short FFQ designed to measure changes in the intake of fruit and vegetable in a Mexican school population where the consumption is only of $34 \%$ [20].

The results on validation average indicate that overall the two methods (FFQ and 24HR) give similar results $(r=0.69)$. This is based on a good correlation between total fruit and vegetable intake measured by both methods. After 6 month of intervention, the reproducibility on total consumption of fruit and vegetable of the questionnaire was 0.73 . According to these results the magnitude of association is high for both validation and reproducibility.

Our food frequency questionnaire seems therefore less suitable for measuring vegetable and fruit consumption alone than combined fruit and vegetable intake. In fact, the weakest correlations were obtained from consumption of fruit. Other research studies have obtained correlations slightly higher for consumption of only fruit than the obtained in our work [2124]. There are possible explanations for this level of validity for fruit. Schoolchildren might eat fruit less frequently or by season which can lead to a lower validity for this variable. Otherwise, is possible to require more $24 \mathrm{HR}$ to be applied for a more precise estimation of fruit intake. We think that it could be because the list of food items in the present study was kept short too (36 items). That is why in this FFQ some open questions have been added to know if schoolchildren have consumed a fruit or vegetable not listed on it. In general, the correlations observed in the literature ranged from 0.5 to 0.8 ; this studio has similar correlations except the one for consumption of only fruit [22-28].

On the other hand, with the Bland-Altman test, the validation was confirmed by observing consistent distribution of points for the control group when the total consumption of fruit and vegetable obtained by the $24 \mathrm{H}$ recall was compared with that of FFQ1. Moreover, the distribution of points behaved differently when the difference in the consumption of fruits and vegetables of FFQ1 against FFQ2 in the intervention group was plotted; here, most of the points were distributed at the bottom of the chart, which was expected because the consumption of fruits and vegetables was promoted and therefore the difference between consumption by FFQ1 and FFQ2 turned to the most schoolchildren in a negative value. These results indicate that the questionnaire is reproducible to measure changes in consumption of fruits and vegetables in schoolchildren.

Another result that confirmed reproducibility of the food frequency questionnaire was the cross tabulation, which ranks individuals according to fruit and vegetable intake, when compared the FFQ1 vs. FFQ2. The distribution of fruit and vegetable intake was acceptable to be divided into equal tertiles, so it may be consider reproducible to assess changes in consumption over time.

Mexico has a food frequency questionnaire made with 130 foods classified into 16 food groups to estimate the usual diet in Mexican schoolchildren aged 5 to 11 years designed by the
National Health Survey (validation is not published). However, is not exclusive to evaluate the consumption of fruits and vegetables, and its application takes longer [29]. In addition, the Mexican FFQ includes fruits and vegetables commonly consumed in the south and center of the country, but little or no consumed in the northwest region. For example, fried banana, crystallized or dried fruit, fruit in syrup, chayote, etc. and in turn discarded fruits and vegetables commonly consumed by schoolchildren in the northwest. Researchers in the area of social nutrition, considered inappropriate to include unusual food consumption, because it would fall into a false response (e.g., shame can lead them to say regularly consuming such foods) and consequently an overestimation of consumption of such food. In this sense, there are two important advantages offered by our questionnaire. First, the exclusivity to estimate the consumption of fruit and vegetable and therefore an application time of only 15 minutes, plus the included foods are culturally consumed by schoolchildren from northwest Mexico. Furthermore, several studies have shown that the intra- and inter-subject variation decreases when information is recorded by frequency consumption than by other dietary method [30]. A limitation of this study might be the relatively small sample size. However, even so, this sample size is most likely to be sufficient, given the positive results found.

Finally, due to the overweight and obesity epidemic facing schoolchildren (up 34.4\%) in Mexico, which is the highest in the world [20]; this questionnaire will be useful for studies and intervention programs for promoting consumption fruit and vegetable in the schools in the short and medium term.

\section{Conclusions}

This food frequency questionnaire has validity and reproducibility to evaluate intake of fruit and vegetable in schoolchildren from northwest Mexico, and can be used to evaluate changes in fruit and vegetable intake on intervention programs, specially aimed to increase the consumption of such food. Due to this FFQ has open questions about the consumption of fruits and vegetables, it has the possibility of increase the quantity of items, this fact will improve the estimation of habitual intake of fruit and vegetables in schoolchildren.

\section{References}

[1] Organización Mundial de la Salud (OMS)/Organización de las Naciones Unidas para la Agricultura y la Alimentación (FAO). Un marco para la promoción de frutas y verduras a nivel nacional. Ginebra: OMS 2005 www.who.int/entity/dietphysicalactivity/reportSP20final.pdf.

[2] World Health Organization (WHO) /Food and Agriculture Organization of the United Nations (FAO). Diet, nutrition and the prevention of chronic diseases. Geneve: WHO; 2003 (Technical Reports Series; NO 916). http://www.who.int/hpr/NPH/does/whofaoexpertreport.pdf. 
[3] Organización Mundial de la Salud. Estrategia mundial sobre régimen alimentario, actividad física y salud. Fomento del consumo mundial de frutas y verduras. OMS, 2012. http://www.who.int/dietphysicalactivity/fruit/es/index1.html.

[4] Ramirez I, Rivera J, Ponce X, Hernández M. Fruit and vegetable intake in the Mexican population: results from the Mexican National Health and Nutrition Survey 2006. Salud Publica Mex 2009; 51 (suppl 4): S574-S585.

[5] Secretaría de Salud. Acuerdo Nacional para la Salud Alimentaria. Estrategia contra el sobrepeso y la obesidad. México: SSa, 2010 [accessed Junio 20, 2016]. Available at: www.promocion.salud.gob.mx

[6] Alejandra Jiménez-Aguilar, Elsa Berenice Gaona-Pineda, Fabiola Mejía-Rodríguez, Luz María Gómez-Acosta, Ignacio Méndez-Gómez Humarán, Mario Flores-Aldana. Consumption of fruits and vegetables and health status of Mexican children from the National Health and Nutrition Survey 2012. Salud Pública Méx 2014; Vol. 56 (sup 2): 103-112.

[7] Willett W C. Nutritional Epidemiology. 2a ed. Nueva York: Oxford University Press 1998.

[8] Ramirez-Silva, I., Rivera, J., Ponce, X., \& Hernández-Ávila, M. (2009). Consumo de frutas y verduras en la población mexicana: resultados de la Encuesta Nacional de Salud y Nutrición 2006. Salud Pública, 574-585.

[9] Plesko M, Cotugna N, Aljadir L. Usefulness of a brief fruit and vegetable FFQ in a college population. Am J Health Behav 2000; $24: 201-8$.

[10] Block G, Gillespie C, Rosenbaum E H, et al. A rapid food screener to assess fat and fruit and vegetable intake. Am J Prev Med 2000; 18: 284-8.

[11] Field A E, Colditz G A, Fox M K, et al. Comparison of 4 questionnaires for assessment of fruit and vegetable intake. Am J Public Health 1998; 88: 1216-18.

[12] Serdula M, Coates R, Byers T, et al. Evaluation of a brief telephone questionnaire to estimate fruit and vegetable consumption in diverse study populations. Epidemiology 1993; 4: $455-463$.

[13] Thompson F E, Kipnis V, Subar A F, et al. Evaluation of 2 brief instruments and a food frequency questionnaire to estimate daily number of servings of fruits and vegetables. Am J Clin Nutr 2000; 71: 1503-10.

[14] Moron C, Viteri F. Update on common indicators of nutritional status: food access, food consumption, and biochemical measures of iron and anemia. Nutrition Reviews. 2009; 67: 31-35.

[15] Block G, Hartman A M, Dresser C M, Carroll M D, Gannon J, Gardner L. A data-based approach to diet questionnaire design and testing. Am J Epidemiol 1986; 124: 453-469.

[16] S M Krebs-Smith, A Cook, A F Subar, L Cleveland, and J Friday. US adults' fruit and vegetable intakes, 1989 to 1991: a revised baseline for the Healthy People 2000 objective. American Journal of Public Health December 1995: Vol. 85, No. 12, pp. 1623-1629. doi: 10.2105/AJPH.85.12.1623.

[17] OMS (Organización Mundial de la Salud). WHO AnthroPlus for Personal Computers Manual. Software for assessing growth of the world's children and adolescents. Ginebra: Organización Mundial de la Salud, 2009.
[18] SPSS INCORPORATION, 2016. SPSS for Windows. Statistical Package for the Social Sciences. Release 19.0. Chicago: SPSS Inc.

[19] Landis J R, Koch G G The measurement of observer agreement for categorical data Biometrics 1977; 33: 159-174.

[20] Encuesta Nacional de Salud y Nutrición (ENSANUT). Resultados nacionales 2012. Instituto Nacional de Salud Pública/Secretaría de Salud. México, 2012.

[21] Alan R. Kristal, Nancy C. Vizenor, Ruth E. Patterson, Marian L. Neuhouser, Ann L. Shattuck, and Dale McLerran. Precision and Bias of Food Frequency-based Measures of Fruit and Vegetable Intakes. Cancer Epidemiology, Biomarkers \& Prevention. Vol. 9, 939-944, September 2000.

[22] Rik P. Bogers, Patricia van Assema, Arnold D. M. Kester, Klaas R. Westerterp, and Pieter C. Dagneli. Reproducibility, Validity, and Responsiveness to Change of a Short Questionnaire for Measuring Fruit and Vegetable Intake. American Journal of Epidemiology. 2004. Vol. 159, No. 9; DOI: 10.1093/aje/kwh123.

[23] Andersen LF, Johansson L, Solvoll K. Usefulness of a short food frequency questionnaire for screening of low intake of fruit and vegetable and for intake of fat. Eur J Public Health. 2002; 12: $208-13$.

[24] Pouya Saeedi, Sheila A. Skeaff, Jyh Eiin Wong and Paula M. L. Skidmore. Reproducibility and Relative Validity of a Short Food Frequency Questionnaire in 9-10 Year-Old Children. Nutrients 2016, 8 (5), 271; doi: 10.3390/nu8050271.

[25] Plaete J, De Bourdeaudhuij I, Crombez G, Steenhuyzen S, Dejaegere L, Vanhauwaert E, etal. (2016) The Reliability and Validity of Short Online Questionnaires to Measure Fruit and Vegetable Intake in Adults: The Fruit Test and Vegetable Test. PLoSONE 11 (7): e0159834. doi: 10.1371/journal. pone. 0159834 .

[26] Vuholm, Stine; Lorenzen, Janne K.; Kristensen, Mette. Relative validity and reproducibility of a food frequency questionnaire to assess dietary fiber intake in Danish adults. Food \& Nutrition Research, [S.1.], dec. 2014. ISSN 1654-661X. Available at: $<$ http://www.foodandnutritionresearch.net/index.php/fnr/article/ view/24723>. Date accessed: 01 Sep. 2016. doi: http://dx.doi.org/10.3402/fnr.v58.24723.

[27] Hebden L, Kostan E, O'Leary F, Hodge A, Allman-Farinelli M (2013) Validity and Reproducibility of a Food Frequency Questionnaire as a Measure of Recent Dietary Intake in Young Adults. PLoS ONE 8 (9): e75156. doi: 10.1371/journal.pone.0075156.

[28] Kolodziejczyk, Julia K.*; Merchant, Gina ; Norman, Gregory J. Reliability and Validity of Child/Adolescent Food Frequency Questionnaires That Assess Foods and/or Food Groups. Journal of Pediatric Gastroenterology \& Nutrition: July 2012 - Volume 55 - Issue 1 - p 4-13 doi: 10.1097/MPG.0b013e318251550e.

[29] Romero-Martínez M, Shamah-Levy T, Franco-Núñez A, Villalpando S, Cuevas-Nasu L, Gutiérrez JP, RiveraDommarco JA. National Health and Nutrition Survey 2012: design and coverage. Salud Publica Mex 2013; 55 suppl 2: S332-S340.

[30] Ortega MI, Valencia ME. Measuring the intake of foods and nutrients of marginal populations. Public Health Nutrition. Vol 5 (6A), 907-910. 2002. 